\title{
Perspective
}

PERSPECTIVE Actualité en histoire de l'art

1 | 2007

Antiquité/Moyen Âge

\section{L'architecture gothique et ses enjeux nationaux en Europe après la Seconde Guerre mondiale}

Points de vue de Philippe Araguas, Klára Benešovská, Thomas Coomans, Peter Draper, Francesca Español, Bruno Klein, avec Dany Sandron

Philippe Araguas, Klára Benešovská, Thomas Coomans, Peter Draper, Francesca Español, Bruno Klein et Dany Sandron

\section{OpenEdition}

Journals

Édition électronique

URL : http://journals.openedition.org/perspective/3743

DOI : $10.4000 /$ perspective. 3743

ISSN : 2269-7721

Éditeur

Institut national d'histoire de l'art

Édition imprimée

Date de publication : 31 mars 2007

Pagination : 74-98

ISSN : 1777-7852

Référence électronique

Philippe Araguas, Klára Benešovská, Thomas Coomans, Peter Draper, Francesca Español, Bruno Klein et Dany Sandron, «L'architecture gothique et ses enjeux nationaux en Europe après la Seconde Guerre mondiale », Perspective [En ligne], 1 | 2007, mis en ligne le 31 mars 2018, consulté le 01 octobre 2020. URL : http://journals.openedition.org/perspective/3743; DOI : https://doi.org/10.4000/perspective. 3743

Ce document a été généré automatiquement le 1 octobre 2020. 


\section{L'architecture gothique et ses enjeux nationaux en Europe après la Seconde Guerre mondiale}

Points de vue de Philippe Araguas, Klára Benešovská, Thomas Coomans, Peter Draper, Francesca Español, Bruno Klein, avec Dany Sandron

Philippe Araguas, Klára Benešovská, Thomas Coomans, Peter Draper, Francesca Español, Bruno Klein et Dany Sandron

1 Gothique : le qualificatif utilisé pour désigner l'art qui s'étendit en Europe du XII ${ }^{\mathrm{e}}$ au début $\mathrm{du} x \mathrm{xvI}^{\mathrm{e}}$ siècle a longtemps cristallisé bien des enjeux nationaux. Mais pour la seconde moitié du $\mathrm{Xx}^{\mathrm{e}}$ siècle, s'interroger sur cet aspect de l'historiographie ne revient pas à chercher les facteurs de permanence signalant une certaine forme de continuité avec le demi-siècle précédent marqué par les crispations nationalistes. C'est plutôt envisager de manière dépassionnée certaines spécificités des approches de la question suivant les différents pays, pour mieux cerner comment elles peuvent servir l'ensemble de la communauté des chercheurs, lesquels nouent individuellement des contacts audelà des limites de leur pays d'origine.

2 Les réponses apportées par les six collègues européens contactés mettent en évidence bien des spécificités qui montrent que la dimension nationale ou régionale de la recherche dans ce secteur reste vivace. Au-delà d'une improbable homogénéité, elles signalent également certaines convergences et le fort potentiel que recèle l'architecture gothique qui peut à bon droit, au propre comme au figuré, être présentée comme un élément-clef de la construction européenne. Comment, sinon à l'échelle du continent, comprendre un art qui s'y est déployé largement à partir $\mathrm{du}_{\mathrm{XI}}^{\mathrm{e}}$ siècle jusqu'à une date avancée $\mathrm{du} \mathrm{XVI}^{\mathrm{e}}$ siècle quand ce n'est pas plus tard, sans parler de la vitalité du néo-gothique qui par certains côtés ramène au cadre national ?

3 C'est celui-ci qui a fixé les limites des vastes opérations d'inventaires sur lesquels les recherches se fondent depuis le XIX ${ }^{e}$ siècle et qui se poursuivent encore actuellement selon des modalités variées. Toutefois, les vicissitudes politiques, l'évolution de la législation ont dans certains pays mis au premier plan des entités territoriales plus 
typées. C'est un des aspects de l'Europe des régions qu'il ne faut pas négliger pour apprécier les tendances historiographiques récentes, encourageantes ou contestables selon les cas. Le dynamisme de l'Espagne à mettre en valeur son patrimoine dans le cadre des gouvernements autonomes des régions peut être opposé à certaines crispations régionales dénoncées pour la Belgique actuelle.

4 À l'exception notable des pays de l'Est isolés jusqu'en 1990 sous le joug de régimes autoritaires, il ressort des réponses fournies que les recherches sur l'architecture gothique depuis la Seconde Guerre mondiale ont prospéré sur la lancée des générations antérieures, avec une relative autonomie à l'égard de la conjoncture, qu'il s'agisse de la reconstruction des zones sinistrées, de la guerre froide ou des alternances politiques à l'ouest. On peut y voir une preuve manifeste de la liberté intellectuelle des chercheurs, mais ce sentiment se mêle parfois au regret de n'avoir pas été davantage associés à la réflexion sur les travaux de restauration et de reconstruction, dans le souci de dépasser cette récurrente dichotomie entre recherche et action sur le terrain. C'est un point qu'il faut garder en mémoire à l'heure où pression immobilière et tourisme de masse pèsent plus que jamais sur le sort du patrimoine. Nos collègues des pays de l'ancien bloc de l'Est confrontés plus récemment à ces phénomènes, parfois insidieux, toujours dévastateurs, s'alarment à juste titre.

5 La recherche fondamentale, si elle s'appuie sur les traditions nationales détaillées dans les pages qui suivent, ne peut échapper à la sclérose si elle ne s'ouvre sur l'extérieur. Qui ne voit que les traditions, positiviste en France, spéculative dans le monde germanique, pragmatique en Angleterre, pour caricaturer sauvagement, doivent s'interpénétrer comme de surcroît intégrer les autres formes de création artistique et se frotter aux autres domaines de la recherche historique? Soulignons à titre d'exemple combien la recherche allemande a profité et fait profiter la communauté scientifique en multipliant les investigations dans divers pays d'Europe, suivant d'ailleurs une longue tradition remontant au XIX siècle, en France, en Angleterre, en Italie, et plus récemment en Espagne et à nouveau en Europe centrale.

6 L'apport extra-européen à l'historiographie du gothique vient avant tout d'Amérique $\mathrm{du}$ nord, nourrie de l'enseignement et des travaux des spécialistes qui y émigrèrent à partir des années 1930. Il est symptomatique que la grande synthèse sur la notion de gothique ait été publiée à Princeton sous la plume d'un universitaire d'origine allemande, Paul Frankl (The Gothic. Literary Sources and Interpretations through Eight Centuries, Princeton, 1960). Le célèbre essai d'Erwin Panofsky (Architecture gothique et pensée scolastique), issu d'une conférence donnée à Latrobe en 1950, quelles que soient les réserves qu'il a pu susciter, reste l'un des textes les plus stimulants qui soit dans notre domaine.

7 Le regard extérieur est naturellement toujours porteur de renouveau, comme ceux de Marvin Trachtenberg qui interroge la notion de gothique italien ${ }^{1}$, de Caroline Bruzelius qui prend en compte les phénomènes édilitaires à Naples ${ }^{2}$, ou encore les travaux de Christian Freigang sur le gothique méridional, en Languedoc en particulier ${ }^{3}$, qui a su s'affranchir des a priori "jacobins" dont l'érudition française ne parvenait pas à se défaire quand elle continuait à donner, contre toute évidence, une interprétation politique - royale - à la diffusion du gothique rayonnant dans le sud du royaume au dernier tiers du XIII ${ }^{e}$ siècle.

8 Cette activité remarquable des chercheurs étrangers n'a malheureusement pas toujours la diffusion escomptée, en raison de la rareté des traductions qui permettent seules 
d'élargir l'audience des travaux : la décision de traduire en castillan l'ouvrage allemand d'Henrik Karge sur la cathédrale de Burgos ${ }^{4}$ est une initiative trop rare qu'on souhaiterait voir étendue pour d'autres enquêtes aussi approfondies.

De l'avis unanime, le renouvellement des approches de l'architecture gothique vient moins des contributions ponctuelles dans une région donnée, toujours utiles cependant, de tel ou tel chercheur étranger, que de la prise en compte des avancées de la communauté internationale et des applications qu'elles peuvent connaître ailleurs. L'ouverture à d'autres domaines de la recherche historique, comme la société dans le livre de Lindy Garant ${ }^{5}$, est le plus fort garant d'un renouvellement des approches. De ce point de vue, la révolution technologique de l'internet, qui permet un accès rapide à des bases de données toujours plus nombreuses et étoffées ainsi qu'à une littérature spécialisée, est présentée unanimement comme la principale donnée nouvelle qui devrait abaisser les frontières, politiques ou intellectuelles, pour le plus grand profit de la recherche.

D.S.

DANY SANDRON. L'historiographie de l'architecture gothique dans la seconde moitié du xx siècle est naturellement le résultat de la convergence de plusieurs composantes, anciennes ou nouvelles. Quel est le poids des traditions académiques nationales remontant au xix siècle (y compris le rôle des sociétés savantes), des institutions patrimoniales d'État (protection, entretien et inventaire des monuments) dans l'historiographie du domaine depuis un demi-siècle?

Peter Draper. Les historiens de la période d'après-guerre ont hérité du $\mathrm{XIX}^{\mathrm{e}}$ siècle une solide tradition pragmatique, combinaison d'analyse archéologique minutieuse et de recherche documentaire, tradition inaugurée par Robert Willis vers 1840-1860 et qu'illustrent ses remarquables études sur les principaux édifices médiévaux, lesquelles sont restées la base de tous les travaux qui ont suivi depuis. Son successeur le plus distingué fut John Bilson (1856-1943) dont les travaux se signalaient par un plus grand intérêt accordé à l'architecture continentale, celle du nord de la France en particulier. À côté de cette tradition archéologique, de nombreuses recherches étaient consacrées à l'histoire liturgique (notamment dans les volumes de la Bradshaw Society) et à l'agencement liturgique des édifices ${ }^{6}$. La plupart de ces travaux paraissaient dans les journaux des principales sociétés savantes, notamment dans le Journal of the British Archeological Association, dans l'Archeological Journal (publication du Royal Archeological Institute), l'Antiquaries Journal et Archaeologia (les bulletins de la Society of Antiquaries), ainsi que dans les publications des nombreuses sociétés archéologiques locales. Ces sociétés nationales et locales ont conservé leur rôle après-guerre. Au cours de la même période, signalons l'importance des guides consacrés à certains monuments (notamment aux ruines monastiques), publiés par le Ministry of Works (l'actuel English Heritage), les notices relatives aux édifices dans les pages du Victoria County History, et tout particulièrement les volumes de la Royal Commission on Historical Monuments ([RCHME], Angleterre et Écosse). À l'origine, la RCHME devait procéder à la recension et à l'inventaire des monuments historiques antérieurs à 1714, mais du fait des lenteurs de l'entreprise et de son extension continue, on a abandonné l'inventaire pour des recherches thématiques et régionales. Pour la période médiévale, il faut mentionner les deux monographies sur la cathédrale de Salisbury (Cocke et Kidson ${ }^{7} ;$ Brown $^{8}$ ), ainsi que l'étude thématique sur les édifices civils de Pearson ${ }^{9}$. La RCHME a été rattachée à l'English Heritage : ses recherches de grande qualité, ses recueils de publications doivent à présent rivaliser, sur le terrain du financement, avec la promotion du patrimoine populaire. 
D'importantes monographies et études thématiques continuent d'être publiées par l'English Heritage, l'Historic Scotland et la CADW (la « historic environment division » du Pays de Galles) sur les monuments dont ils ont la charge et ces institutions encouragent toujours les études d'histoire de l'architecture médiévale, y compris des recherches techniques comme la dendrochronologie.

Mais l'historiographie de l'architecture gothique a trouvé sa meilleure tribune dans les conférences médiévales annuelles de la British Archeological Association (instituées en 1975) et dans les publications auxquelles elles ont donné lieu. Dans ces volumes, cathédrales, abbayes et églises ont été étudiées de près ainsi que leur environnement, et au cours des dernières années, les chercheurs ont étendu leurs investigations au-delà des îles britanniques pour s'intéresser à des sites comme Rouen, Utrecht, Angers, Mayence et Prague. Tandis que bon nombre de contributions perpétuaient les solides traditions de l'analyse archéologique, de nouvelles approches ont été intégrées et le champ d'enquête s'est élargi pour inclure la décoration, les aménagements du mobilier et de la liturgie, rassemblant ainsi sous une forme inédite des objets dont le $\mathrm{XIX}^{\mathrm{e}}$ siècle avait inauguré l'étude. Les enjeux de cette réintégration des divers éléments de l'architecture gothique ont été explicités et analysés dans un colloque organisé à Toronto, dont les actes ont été publiés dans Artistic integration in Gothic Buildings ${ }^{10}$.

Au cours des années 1950, la Conway Library arrivée au Courtauld Institute en 1932 a constitué, grâce à des donations et aux missions photographiques qu'elle organisait, les plus importantes archives d'architecture gothique du Royaume-Uni. Elle a aussi formé le foyer d'une communauté savante de chercheurs en architecture médiévale, pour la plupart anciens élèves de Peter Kidson, qui fut dans ce domaine le plus influent spécialiste de la période d'après-guerre. Le recueil d'essais publié en son honneur reconnaît la fécondité de son enseignement ${ }^{11}$ et offre un échantillon significatif des diverses approches qui se sont affirmées dans les dernières années du $\mathrm{xx}^{\mathrm{e}}$ siècle. Dans son introduction et les longues notes de bas de page de l'édition de Gothic Architecture de Paul Frankl (2000) revue par Paul Crossley, celui-ci replace Frankl dans son contexte intellectuel et propose une analyse faisant autorité de différentes approches de l'architecture gothique en Europe ${ }^{12}$.

Philippe Araguas. L'histoire de l'architecture gothique, telle qu'elle a été pratiquée dans la deuxième moitié du $\mathrm{xx}^{\mathrm{e}}$ siècle en France, s'inscrit, au moins jusque dans les années 1980, dans la stricte mouvance de l'archéologie monumentale telle qu'elle a été empiriquement définie dans la deuxième moitié $d u$ xix ${ }^{e}$ siècle et plus ou moins théorisée par Camille Enlart ou Jean-Auguste Brutails. En affirmant cela, je n'insinue pas de manière perfide que cette pratique est marquée du sceau de l'archaïsme car, à mon sens L'archéologie du Moyen Âge et ses méthodes publié par Brutails chez Picard en 1900 n'a rien perdu de sa pertinence méthodologique (jusque dans la dénonciation " de l'abus des notions scientifiques en archéologie »). L'atout majeur de l'archéologie monumentale française a été d'associer à l'observation des monuments, sous les angles multiples de la conception de la construction de la structure et du décor illustrée par Viollet-le-Duc, la prise en compte de la documentation historique avec le soin et la rigueur de la méthode de l'École des chartes. Que cette étroite symbiose entre l'esprit chartiste de Quicherat et la pratique du relevé technique "violletleducien " puisse conforter une vision excessivement positiviste des édifices gothiques ne constitue pas à mon sens un handicap, mais une force du discours de 
l'histoire de l'architecture gothique "à la française ", car c'est ce qui donne une assise inébranlable à des constructions plus spéculatives sur lesquelles je reviendrai ultérieurement.

C'est dans le sillage méthodologique de Brutails que s'inscrivent les grandes thèses sur l'architecture gothique de la deuxième moitié $\mathrm{du} \mathrm{xx}^{\mathrm{e}}$ siècle, qui sont toutes peu ou prou marquées par l'empreinte "chartiste». Le principal atout de cette tradition historiographique réside dans les centaines de notices, articles, communications dispersées dans les publications des sociétés savantes, à commencer par celles de la Société française d'archéologie : Bulletin Monumental et actes des Congrès archéologiques de France. Ce travail fantastique des sociétés savantes, toujours actives en dépit de leur grand âge et du vieillissement de leurs cadres, laisse loin en retrait l'action des institutions patrimoniales. Si l'administration des Monuments historiques, qui fut si importante dans l'éclosion de la discipline, s'est impliquée dans l'histoire de l'architecture gothique, ce n'est que par l'investissement collectif ou individuel de certains des architectes des divers services plus ou moins affiliés à cette instance que par des initiatives propres.

La création en 1964 de l'Inventaire général qui s'appuyait originellement sur le réseau encore puissant des sociétés savantes pouvait laisser espérer un renouvellement qualitatif et quantitatif des études sur l'histoire de l'architecture ; le travail de réflexion méthodologique et théorique mené dans ce cadre constitue incontestablement un acquis fondamental pour l'histoire de l'architecture, mais il faut bien reconnaître que dans le domaine de l'architecture gothique les apports sont relativement minces et noyés dans une production scientifique qui, devant prendre en compte le patrimoine depuis la petite cuillère jusqu'à la cathédrale a, délibérément, privilégié la petite cuillère. Quant au service de l'archéologie, sa définition trop marquée par le «fouillocentrisme » l'écartait pour des décennies de l'histoire de l'architecture.

Cette atteinte portée par l'archéologie de fouille à l'unicité de l'archéologie médiévale avait eu son brûlot, en dépit de l'incontestable largeur de vue de son auteur, avec le Manuel d'archéologie médiévale de Michel de Boüard (1975) qui a isolé pour une longue période les archéologues «monumentaux » des « sédimentaristes ». Ce n'est que très récemment que se réduit, autour de l'archéologie "du bâti» cette fracture méthodologique confortée par l'esprit de clocher et les pratiques corporatistes de tout crin. On est en droit d'attendre beaucoup d'expériences comme celles menées par le Centre d'études médiévales d'Auxerre qui unifie l'expérience séculaire de l'archéologie monumentale des sociétés savantes à celle plus récente de l'archéologie médiévale "sédimentaire " et "du bâti ", en intégrant une archéométrie dont les actions parfois dévastatrices (vieillissement soudain du donjon de Loches, rajeunissement spectaculaire des églises wisigothiques espagnoles) n'ont semble-t-il pas encore atteint les études sur l'architecture gothique.

Thomas Coomans. Avant 1914, l'historiographie gothique a longtemps été nationale ${ }^{13}$ et fut, à partir de 1862, liée au mouvement des Écoles Saint-Luc. Celles-ci, d'obédience ultramontaine et davantage marquées par la doctrine morale de Pugin que par le rationalisme de Viollet-le-Duc, développèrent l'idée d'un style gothique national et catholique, et formèrent plusieurs générations d'architectes, d'artistes et de restaurateurs. La Commission royale des Monuments, fondée en 1835, ainsi que les 
sociétés archéologiques qui fleurirent dans toutes les villes dès avant 1850 et organisèrent les Congrès de la Fédération archéologique et historique de Belgique à partir de 1885, eurent un rôle déterminant tant pour la restauration de la plupart des grands monuments gothiques que pour le développement du mouvement archéologique à partir des années 1860. Les théories sur les écoles régionales romanes (scaldienne et mosane) et gothiques (scaldienne, mosane et brabançonne) se développèrent à partir des années 1890 , l'intérêt se portant non seulement sur les monuments, mais aussi sur les maisons urbaines et l'architecture vernaculaire.

Les destructions de la Première Guerre mondiale sur le patrimoine architectural belge, y compris gothique, furent considérables (Ypres, Dinant, Louvain, Anvers, Malines, etc). Dans les années 1920, toute l'énergie fut absorbée par la reconstruction qui fut rapide, sur fond de débat entre tradition et modernité, conservatisme et modernisme. Les années 1930 virent, en matière d'historiographie gothique, deux phénomènes à forte connotation nationaliste. D'une part, Stan Leurs, professeur à l'Université de Gand, développa la théorie nationaliste flamande du «style gothique thiois " (thiois/diets = espace culturel germano-hollando-flamand), dans de nombreuses publications, y compris en allemand pendant la guerre. D'autre part, deux écoles s'attelèrent à résoudre la question de l'origine du style gothique en Brabant: les chercheurs de l'Université de Gand, devenue flamande en 1930, épluchaient les sources écrites, tandis que ceux de Louvain étudiaient les bâtiments. La question de l'origine était cruciale, car elle était identitaire. Le débat se déroula surtout au sein de la classe des beaux-arts de l'Académie royale flamande de Belgique, de 1940 à 1944.

$\mathrm{Au}$ lendemain de la guerre, l'architecture gothique continua de mobiliser, mais dans une perspective régionaliste. Une initiative intéressante fut la réalisation en 1987, par des chercheurs flamands et hollandais, d'une exposition transfrontalière sur les architectes Keldermans, les principaux maîtres d'œuvre du gothique flamboyant brabançon au $\mathrm{XV}^{\mathrm{e}}$ et au début du $\mathrm{xVI}{ }^{\mathrm{e}}$ siècle $^{14}$. Cependant, les tensions communautaires croissantes menèrent à la scission progressive de l'État belge à partir de 1970. Les institutions patrimoniales furent parmi les premières à être scindées : d'abord transférée aux communautés culturelles flamande et wallonne, la compétence sur le patrimoine immobilier échut aux régions flamande, bruxelloise et wallonne en 1989. Depuis lors, chacune développe des programmes très différents, notamment en matière d'inventaire, de protection et de restauration, possède une législation et un budget distincts, et initie des politiques de recherche et de publication spécifiques. L'autonomie est totale, la concertation, inexistante si ce n'est par le détour d'institutions internationales telles que l'ICOMOS.

Bruno Klein. Tout d'abord, il faut se rappeler qu'en Allemagne entre 1945 et 1990, on poursuivit deux chemins différents. Tandis qu'en Allemagne de l'Ouest, la reprise des traditions et des structures d'avant-guerre put se faire de manière quasi immédiate et donc tout aussi problématique -, en RDA le champ de la recherche fit davantage l'objet de restructurations. En tant que phénomène particulier, les impulsions les plus importantes, par rapport à la recherche sur le gothique, étaient notamment issues de l'aile de la conservation du patrimoine (Schubert, Magirius, Krause, Badtstübner), alors que les travaux académiques, à cause de leur orientation idéologique inévitable, n'étaient souvent que d'une valeur très limitée (concernant le rôle de la conservation du patrimoine en RFA, voir la réponse à la deuxième question). 
En Allemagne de l'Est, on misait davantage sur la tradition nationale dans le secteur culturel qu'à l'Ouest où on favorisait plutôt l'intégration au sein de la culture atlantique. Ces caractéristiques valaient aussi bien pour l'architecture contemporaine et les beaux-arts que pour les recherches historiques. De manière particulièrement intense, on s'intéressait à l'architecture gothique française - un fait qui renoue par ailleurs avec des traditions plus anciennes qui remontent pour la plupart au XIX siècle. Pour l'époque après 1945, d'importants travaux en allemand, souvent des monographies, ont été consacrés aux édifices de Besançon, Braine, Carcassonne, Châlons-en-Champagne, Champmol, Chartres, Meaux, Metz, Paris, Reims, Rodez, Rouen, Toulouse, Tours, Troyes et Vincennes - une liste qui ne revendique pas l'exhaustivité. À ces publications s'ajoutent encore l'ouvrage général de Dieter Kimpel et Robert Suckale ${ }^{15}$, ainsi que celui de Willibald Sauerländer ${ }^{16}$.

Entre 1950 et 1975, bien qu'elle semble avoir diminué depuis, c'est la recherche sur l'architecture gothique italienne qui se développe parallèlement à celles sur la France. Maintenant, l'Espagne et l'Angleterre commencent à entrer également en considération. La spécialisation sur l'architecture gothique en France se fait au détriment de l'architecture gothique allemande, quand elle ne témoignait pas de liens privilégiés avec la France. Ainsi, les édifices inspirés par l'architecture française à Trèves, à Marbourg et à Cologne sont-ils mieux connus et plus présents dans le discours commun de l'histoire de l'architecture que de nombreux monuments de la fin du Moyen Âge (il y a donc un vaste champ pour la recherche à venir...).

Les raisons de cette évolution semblent être multiples: la volonté de s'orienter suivant le gothique français, soi-disant "plus systématique "; le rejet du gothique allemand en tant que "cas particulier», un concept (Sondergotik) alourdi par le contexte ethnique et nationaliste; la séparation de l'Allemagne qui rendait les recherches approfondies du corpus des monuments impossibles avant 1990. Enfin, il ne faut pas non plus oublier que l'absence d'études sur le gothique après 1270 est un phénomène commun aux recherches allemande et française - un élément qui souligne l'impact des traditions universitaires plus anciennes. Font exception, depuis toujours, les constructions des Parler, avant tout à Schwäbisch Gmünd, à Prague et à Vienne, et l'architecture qui en dérive. Les deux grandes expositions, l'une sur les Parler et l'autre sur Prague ${ }^{17}$ ont démontré de façon impressionnante que cet art, grâce à sa dimension européenne, bénéficia d'un intérêt particulier pendant les périodes de l'après-guerre et d'après la chute du mur.

À Norbert Nußbaum revient le mérite d'avoir toujours souligné l'importance des autres exemples de l'architecture du gothique tardif ${ }^{18}$. Ces dernières années, les recherches dans ce domaine ont augmenté, sans renouveler le lien avec les problématiques de l'avant-guerre. Les impulsions majeures pour la recherche proviennent des milieux de la conservation des monuments. À ce titre, on peut évoquer les fouilles, réalisées dans le cadre de la reconstruction des cathédrales de Cologne et de Trèves, qui ont permis de reconstituer l'histoire de ces édifices jusqu'à l'Antiquité. Les études récentes de la cathédrale de Ratisbonne prennent indirectement le relai en proposant une approche complexe de la pratique et de la technique des constructions médiévales. Cela est également valable pour la publication des recherches en architecture qui étaient nécessaires et à nouveau possibles après la réunification. 
Il faudrait encore souligner les travaux qui ont été réalisés sur la polychromie de l'architecture médiévale, comme ceux de Jürgen Michler.

Klára Benešovská. Avant de répondre à cette question, j'aimerais préciser deux choses : parler de l'historiographie de l'architecture gothique suppose qu'on prendra en compte l'historiographie du Moyen Âge dans son ensemble. Quant à l'Europe centrale et orientale (Ostmitteleuropa) pour l'historiographie de l'architecture gothique, elle correspond au territoire contenu à l'intérieur des frontières des pays de l'ancienne monarchie des Habsbourg autour de 1900. Ces pays ont pour leur histoire de l'art une racine commune, l'école de Vienne, tandis que pour les territoires de l'Allemagne de l'Est et de la Pologne occidentale l'école de Berlin dominait. Enfin, en raison de l'histoire particulière des pays issus de l'Empire austrohongrois qui sont passés après 1945 dans le bloc communiste, je me permettrais d'insister sur la phase «nationale » des années 1918-1939 dans cette réponse.

Le poids des traditions académiques nationales remontant au $\mathrm{XIX}^{\mathrm{e}}$ siècle dans l'historiographie est absolument fondamental. La médiévistique (historiographie de l'architecture gothique incluse) forgée dans le creuset de l'école de Vienne, en est restée prisonnière, méthodologiquement, jusqu'aux années 1950. De plus, l'institutionnalisation de la discipline s'est développée parallèlement à la naissance des institutions patrimoniales d'État (1850: fondation de la k.k.Zentralkomission für die Erforschung und Erhaltung der Kunst- und historischen Denkmäler; 1852 : nomination de Rudolf Eitelberger von Edelberg comme professeur d'histoire de l'art; 1854: naissance de l'Institut für österreichische Geschichtsforschung - suivant le modèle de l'École des chartes -, auquel est rattaché le département d'Histoire de l'art un peu plus tard). R. Eitelberger avec Gustav Adolph von Heider, président de 1866 à 1873 de l'Académie des Arts à Vienne, fut aussi le fondateur de la première topographie des monuments médiévaux du territoire de l'Empire autrichien (Mittelalterliche Kunstdenkmäler des österreichischen Kaiserstaates, 1858-1860) et de l'édition des sources (Quellenschriften für Kunstgeschichte und Kunsttechnik des Mittelalters und der Renaissance, 1871-1882 [Sources pour l'histoire de l'art et des techniques artistiques du Moyen Âge et de la Renaissance]). En 1873, Moriz Thausing (1835-1884) organise le premier Congrès international d'histoire de l'art à Vienne, considéré comme le fondateur de la discipline ${ }^{19}$.

Comme Ján Bakos le fait remarquer à juste titre ${ }^{20}$, ces institutions nouvellement créées devaient légitimer la restauration de la puissance de l'aristocratie après la révolution bourgeoise de 1848 et contribuer à la centralisation de l'Empire des Habsbourg par l'idée d'un héritage culturel transnational. Cette nouvelle idéologie était censée aider à surmonter les tensions nationalistes et sociales qui traversaient la monarchie multinationale restaurée après 1848 .

L'école de Vienne devait avant tout former des spécialistes appelés à travailler dans les musées, archives et institutions patrimoniales. Les historiens d'art devaient en tant qu'experts de l'État devenir les piliers de la bureaucratie de l'Empire. La protection de l'héritage culturel servait de prétexte pour promouvoir le patriotisme transnational à l'aide des outils de la science. Aussi les protagonistes de ce qu'on appelle "première école de Vienne ", Moriz Thausing, Franz Wickhoff, Alois Riegl, Julius von Schlosser, commencèrent-ils alors leurs carrières dans les musées ou dans la protection des monuments ${ }^{21}$. 
Il faut reconnaître que ce patriotisme a, pour ces régions, sauvé l'histoire de l'art du nationalisme qui à l'époque marquait la discipline en Allemagne. Riegl a ainsi critiqué la reconstruction à la manière romantique des châteaux en Allemagne (architecte : Bodo Ebhardt), ainsi que Georg Dehio pour sa direction nationale de la protection des monuments ${ }^{22}$. Ce patriotisme autrichien, démocratique et transnational, est patent après la Première Guerre mondiale avec la naissance des États nouveaux (Tchécoslovaquie, Hongrie, Autriche, Roumanie, Yougoslavie). Les élèves de l'école de Vienne ont occupé les principaux postes institutionnels et ont cherché à libérer la discipline de sa dépendance envers l'histoire de la culture pour la transformer en une science "exacte». Mais ils étaient en même temps tenus de défendre l'existence spécifique de l'art et du patrimoine «national» des nouveaux États. Il faut souligner qu'ils ne sont jamais tombés dans le piège d'un nationalisme irrationnel et raciste. On peut citer l'exemple de la réaction de Vojtech Birnbaum, élève de Wickhoff et de Riegl, professeur à l'université de Prague (1919-1934), à un débat remontant déjà au XIX ${ }^{e}$ siècle. S'y opposaient le professeur de l'université allemande de Prague Josef Neuwirth ${ }^{23}$, tenant d'un art médiéval dans les pays tchèques considéré comme un rejeton de l'art germanique, créé par les artistes allemands, et les chercheurs tchèques de la génération plus ancienne qui au contraire avaient cherché à présenter cet art comme le produit d'une créativité nationale, à l'évolution indépendante et autochtone. Fidèle à l'esprit de l'école de Vienne, Birnbaum a renvoyé dos à dos ces deux points de vue pour défendre le caractère transnational de l'évolution des formes ${ }^{24}$.

Il est vrai que les disciples de l'école de Vienne, pris par les problèmes pratiques des institutions qu'ils dirigeaient et qu'ils avaient aidé à fonder dans les pays créés après la chute de l'Empire, avaient peu de raisons de réfléchir à de nouvelles méthodes en histoire de l'art. Ils n'ont pas eu non plus suffisamment de temps : vingt ans après la naissance de ces nouveaux États, leur existence était déjà menacée. À l'intérieur des nouvelles frontières «Böhmen und Mähren » du Reich allemand, après la fermeture des universités tchèques, seuls des historiens allemands (Karl Maria Swoboda, Otto Kletzl, Erich Bachmann ${ }^{25}$ ) ont publié sur l'art médiéval gothique du territoire de l'ancienne Tchécoslovaquie, présenté en tant qu'art allemand.

Francesca Español. Dans le contexte hispanique, l'architecture gothique a longtemps été l'apanage des architectes, comme en témoigne l'historiographie : Puig i Cadafalch, Lampérez, Chueca Goitia, Martinell, Bassegoda, etc. Ce fut significativement l'un d'eux, Torres Balbás, qui signa le texte traitant de cette question publié en 1952 dans la collection "Ars Hispaniae ». Depuis un certain temps, cette tendance s'est inversée. Les recherches sur l'architecture gothique sont menées par des historiens d'art liés à l'université et des spécialistes de la discipline qui occupent des postes administratifs en rapport avec la conservation du patrimoine, domaine qui, en Espagne, dépend des Communautés Autonomes et des Députations provinciales.

Cette spécialisation a été déterminante sur le plan historiographique. Les études portant sur des constructions d'époque gothique se sont poursuivies selon les directives figurant dans les travaux des architectes du début du $x^{e}$ siècle cités plus haut. On a décrit les méthodes, compilé les données connues sur les processus de réalisation en notant leur chronologie probable et comparé équipes et structures afin d'en dégager ou souligner les caractéristiques originales. La documentation (d'une grande richesse dans certains secteurs) a également facilité les études sur les 
architectes tels que Guillem Sagrera, Juan Guas, Gil de Hontañón etc. Cette perspective est sous-jacente dans les études publiées entre 1950 et 2000 dont la valeur scienti-fique est à signaler.

$\mathrm{Si}$, comme nous l'avons souligné, le poids des architectes dans l'historiographie de la deuxième moitié $\mathrm{du} \mathrm{xx}^{\mathrm{e}}$ siècle a diminué, leur prééminence (parfois avec celle des archéo-logues) sur l'historien d'art se maintient toutefois dans certains dossiers. En ce sens, leur position à la tête des diverses équipes constituées dans le cadre du « Plan des cathédrales » sous le patronage du Ministère de la culture en 1997, avec pour objectif d'amorcer la restauration des cathédrales espagnoles parmi lesquelles figurent de nombreux ouvrages gothiques (Burgos, Vitoria, Gerone etc.) est significative. Les historiens d'art font partie de ces équipes, mais leur travail s'en tient à la rédaction d'études destinées à alimenter l'historique de la restauration.

Dans les études sur l'architecture gothique, le rôle des sociétés, nées à la fin du XIX siècle ou au cours de la première moitié $d u x^{e}$, est assez insignifiant. Les séances de travail ont cessé dans de nombreux cas et les nombreuses revues qui continuent à être publiées sous leurs auspices acceptent toute étude sans la soumettre à aucune critique. Le résultat est prévisible: les travaux présentant des points de vue innovants - minoritaires - alternent avec des articles qui s'attachent à la description de l'édifice ou avec la présentation d'un document inédit que l'on donne simplement à connaitre. Malheureusement, en matière d'histoire de l'art espagnol, l'absence de débat historiographique s'étend à d'autres domaines et institutions qui ont un rapport avec notre discipline.

Depuis 1950, on continue à publier des inventaires et des catalogues de monuments dans des provinces et des villes avec la même ambition que celle des travaux précurseurs menés depuis 1900, à l'initiative de la Real Academia de Bellas Artes de San Fernando, entre autres par Manuel Gómez Moreno (sur Avila, Salamanque, Zamora, León), José Ramón Mélida (sur Cáceres et Badajoz), José Hernández Díaz et autres (sur Séville), Ricardo del Arco (sur Huesca) ou Juan Ainaud et autres (sur Barcelone). Parmi les quatre volumes de la province de Palencia, le premier parut en 1946 (sous la direction de Rafael Navarro García). Les vingt volumes de la province de Valladolid sont publiés depuis 1960 (en rééditant les volumes épuisés).

Traditionnellement, ces publications relèvent de la compétence d'historiens d'art et d'archéologues, mais les projets les plus récents touchant à l'architectonique sont du ressort des architectes. En 1970 a commencé l'édition de l'Inventaire artistique espagnol par provinces, presque achevée aujourd'hui, et les auteurs des travaux sont essentiellement des universitaires. L'ambition de ce projet éditorial initié par la Direction du patrimoine culturel qui dépend du Ministère de l'éducation et des sciences est bien moindre que celle du Catálogo Monumental de España [Catalogue des monuments d'Espagne] et les résultats scientifiques très inégaux. Dans bon nombre d'entre eux, la description prévaut sur l'analyse et, s'agissant de textes assez courts, la partie consacrée à l'architecture gothique est nécessairement brève et souvent peu exploitable.

Certains catalogues et inventaires relèvent d'autres choix éditoriaux et se détachent de l'ensemble. Ils sont le fait particulièrement des organismes bancaires et des ministères des diverses communautés autonomes auxquelles a échu la responsabilité du patrimoine à la suite des réformes constitutionnelles postfranquistes. C'est le cas 
du Catálogo Monumental de Castilla-León de bienes inmuebles (1995) de Javier Ribera, théoricien de la restauration, ou de ceux que publie la Communauté de Castille-La Manche. Cela a contribué à améliorer la qualité de l'édition (format des ouvrages et apparat graphique). Parfois, grâce à un financement externe, l'Église a elle-même lancé ces projets, comme dans le diocèse de Vitoria, pionnier dans ce domaine, dont le catalogue des monuments en six volumes fut élaboré entre 1967 et 1998 . Beaucoup de ces catalogues se signalent par leur rigueur et leur ampleur, tels ceux de la Communauté autonome de Navarre ou de Cordoue.

Ces catalogues et les inventaires qui compilent l'architecture et les objets mobiliers de villes, provinces et régions, constituent un instrument indispensable dans notre approche et notre évaluation du patrimoine artistique conservé ou perdu à la suite de la guerre civile. Dans le cas des édifices médiévaux et plus concrètement des cathédrales gothiques espagnoles, cette radiographie s'établit également à partir d'une série de colloques monographiques. Ils n'obéissent pas à un plan préétabli mais plusieurs dus à Joaquín Yarza possèdent une certaine unité (sur la cathédrale de Lérida [1991], sur Santo Domingo de la Calzada [2001] et sur León [2003]).

DANY SANDRon. Quel fut l'impact des conflits européens des décennies 1930-1940 et des campagnes de reconstruction qui ont pu suivre?

Francesca Español. Dans le cas de l'Espagne, la guerre civile (1936-1939) a eu le même effet dévastateur que la Seconde Guerre mondiale et a poussé à entreprendre des restaurations architecturales sur tout le territoire par le biais d'un organisme créé en 1938, le Service national des Régions dévastées et des réparations, qui a acquis ultérieurement le rang de Direction générale. Cet instrument très efficace s'est occupé non seulement des bâtiments historiques (pour lesquels ont travaillé presque exclusivement des architectes tels que Fernando Chueca Goitia, César Martinell, Alejandro Ferrant) mais aussi de la reconstruction des villes touchées par la guerre et des bâtiments médiévaux qui se détérioraient depuis le $\mathrm{XIX}^{\mathrm{e}}$ siècle, après avoir été abandonnés par suite de la sécularisation des biens ecclésiastiques (1835) qui a entraîné la ruine de nombreux monastères. Même si certains d'entre eux étaient des historiens de l'architecture, ces architectes des «Régions dévastées » ont entrepris ces restaurations en se guidant sur des critères historiques nourris des présupposés du XIX siècle.

Ainsi cette vaste campagne n'a suscité aucun débat théorique. Le pragmatisme a primé et la documentation issue de ces chantiers le reflète de façon éloquente. Souvent en effet, il n'existe aucune trace écrite des interventions réalisées (à part les documents comptables), et les relevés d'architecture s'avèrent insuffisants pour les évaluer. D'où la difficulté pour l'historien de suivre le déroulement de ces chantiers.

La phase de reconstruction historique menée par les architectes espagnols a duré en Espagne de l'après-guerre civile jusqu'à la fin du $\mathrm{xx}^{\mathrm{e}}$ siècle. Elle comprend donc les premiers temps du franquisme, marqué par le boom touristique, avec la promotion des lieux à dimension historique et de leurs monuments. Entre 1962 et 1972, aux "Paradores de Turismo" [hôtels de luxe] déjà existants s'ajoutent de nombreux autres (pas moins de seize établissements ont été inaugurés en 1972). Installés pour la plupart dans des bâtiments déclarés d'intérêt historico-artistique ou des monuments nationaux, leurs aménagements étaient régis par les codes propres à leur nouvelle fonction touristique. 
Depuis la fin des années 1970, toutefois, la reconstruction historique coexiste avec une autre, de principe opposé - et critique -, qui se nourrit aujourd'hui d'une réflexion théorique sur le rôle qui revient à l'architecte-restaurateur. Le débat s'est ouvert dans d'autres pays européens, mais en Espagne il a été excessivement restreint aux architectes. La faible présence des historiens d'art n'est pas tant due à leur manque d'intérêt pour la question qu'à la situation engendrée par la législation qui impute la responsabilité maximale aux architectes en matière de travaux de restauration. Le fait d'attribuer une telle capacité de décision à ce groupe professionnel empêche bien souvent les apports des disciplines historiques d'être pris en compte.

Bruno Klein. Incontestablement, la Seconde Guerre mondiale a eu des conséquences déterminantes à la fois pour l'architecture et pour la recherche. Pour être plus complet et plus clair, les deux aspects sont ici présentés séparément.

- Architecture : il n'est pas possible de distinguer nettement les retombées de la destruction due à la guerre de celles de la reconstruction qui, dans certains cas, fut aussi dévastatrices.

Il faut d'abord mentionner les pertes du patrimoine original causées par des destructions entières ou partielles. Ces dégâts n'ont jamais pu être réparés entièrement: même après la reconstruction, l'authenticité des édifices restait atteinte. À cela s'ajoute l'aspect particulier qu'en RDA, jusque dans les années 1960, de grandes églises gothiques furent encore détruites pour des raisons idéologiques (par exemple à Dresde et à Leipzig). Par manque de moyens financiers, l'entretien d'autres édifices fut nettement insuffisant.

Les conséquences de la guerre et de la reconstruction furent fatales pour certaines villes historiques. Pour diverses raisons, les paysages urbains fortement caractérisés par des éléments gothiques, comme à Lübeck, Wismar, Stralsund, Greifswald et Regensburg, furent particulièrement endommagés.

Les trente premières années de reconstruction témoignent souvent de réédifications purifiées ou créatrices qui ont aggravé les pertes d'authenticité.

- Recherche : à partir des années 1970, tous ces éléments suscitèrent des critiques qui servirent à une approche des monuments plus nuancée, soutenue par des travaux de recherche. Ainsi, dans les villes, les groupements d'édifices et l'équipement des constructions sont aujourd'hui davantage traités en tant qu'unités; les recherches portèrent sur les liens avec l'histoire et la liturgie, tandis que l'œuvre d'art est moins perçue comme un objet muséal. La reconstruction évoquée ci-dessus eut de lourdes conséquences pour les recherches menées au sein de la conservation du patrimoine.

L'impact de l'époque national-socialiste et de la guerre est resté très sensible au niveau du contenu. Même si le livre Die Entstehung der Kathedrale de Hans Sedlmayr (1950), imprégné de l'idéologie nazie, a pu encore paraitre après la fin de la Seconde Guerre mondiale, la plupart des chercheurs se sont intéressés à une objectivation du contexte socio-historique et de l'histoire des styles (Otto von Simson, Werner Gross). Or cette objectivation allait de pair avec la tendance qui opposait à un présent problématique, marqué par le nazisme, la guerre froide et une modernisation plus générale, l'image idéalisée du Moyen Âge. 
Critiques face à l'idéologie, les travaux de Martin Warnke, Dieter Kimpel et Robert Suckale, ouverts aux analyses plus complexes du contexte historique, ont libéré la recherche gothique allemande, au début des années 1970, de l'approche formelle immanente, et l'ont réintégrée dans le cercle scientifique international. Depuis, Günther Binding et Wolfgang Schöller ont proposé des études de référence sur la technique, l'organisation du chantier et le financement en architecture. Par ailleurs, de nouveaux manuels sont apparus, dont celui de Wolfgang Schenkluhn sur l'architecture des ordres mendiants ${ }^{26}$, les ouvrages de Matthias Untermann sur les constructions cisterciennes (avec une orientation internationale) ${ }^{27}$, ainsi que celui de Norbert Nußbaum sur l'architecture gothique en Allemagne.

Klára Benešovská. Ce ne sont pas seulement les victimes, les ruines et les pertes économiques que la guerre a laissées derrière elle, mais aussi, et avant tout, les blessures psychiques de la société. De plus, le partage politique de l'Europe et la division de celle-ci en deux blocs par le rideau de fer a eu des conséquences considérables sur plusieurs plans.

La destruction des villes historiques en Europe provoqua logiquement un débat tendu sur la protection des monuments. Les positions de Riegl et Dvorak, qui avaient préféré la conservation à la reconstruction ou la réplique, furent écartées. La reconstruction totale de Varsovie, de Gdansk ou des villes allemandes est devenue la question de la restauration de l'identité nationale, symbole de la renaissance culturelle d'après-guerre. En revanche, les imitations et les reconstructions des bâtiments et des églises détruits par la guerre ont aidé à une meilleure compréhension des aspects architectoniques et technologiques et permis la mise en œuvre de méthodes plus scientifiques, appliquées à l'analyse des monuments.

Dans le domaine des sciences humaines, la guerre a aussi laissé des traces, même si cela a été moins spectaculaire. Un exemple emprunté à l'historiographie de l'architecture gothique tchèque en est le livre de Vaclav Mencl ${ }^{28}$ (1905-1978), réaction immédiate aux interprétations nationalistes de l'architecture gothique sur le territoire de la Tchécoslovaquie et à la répression qui eut cours durant les six années de guerre. Les circonstances et les raisons pour lesquelles ce livre a vu le jour ont influencé sa méthode, qui d'un côté faisait la part belle à la tradition de l'école de Vienne (et à Birnbaum) ${ }^{29}$, et de l'autre côté au structuralisme de l'école de Prague (Roman Jakobson, Jan Mukarovsky) et aux écrits de Wilhelm Dilthey. Les résultats de ses analyses pénétrantes, source d'inspiration encore aujourd'hui, sont ici subordonnés à sa volonté de défendre l'existence d'une architecture gothique typiquement tchèque dont la spécificité, d'après l'auteur, serait une approche «sensible » et non rationnelle qui conduit au style du gothique tardif ${ }^{30}$. Le paradoxe de cette "micro-histoire » prise dans la «macro-histoire» de l'Europe de l'Est des années 1948-1955, c'est la fin qu'il a connue : le livre fut condamné par les étudiants (communistes) de l'Université Charles, comme exemple idéologiquement très dangereux ; Mencl dut quitter l'université et faillit terminer ses jours en prison.

La fermeture des frontières, l'omniprésence et l'omnipotence de l'idéologie ont particulièrement isolé l'historiographie de l'architecture gothique dans les pays de l'Est dans les quatre décennies d'après-guerre.

La tradition de Vienne se poursuivit, mais limitée aux aspects formels : les études sur l'architecture gothique se limitaient à la description et à la reconstitution de 
l'évolution des formes, à la lumière des sources - seules méthodes acceptables selon l'idéologie marxiste. Au début des années 1960, la pression se relâcha un peu et favorisa les contacts des chercheurs avec leurs collègues à l'étranger, mais cela prit fin en août 1968 avec l'invasion soviétique. Malgré cela l'activité se concentra sur les monographies des artistes, des bâtiments ou des villes ainsi que sur les synthèses ${ }^{31}$, les contacts non-officiels entre médiévistes de spécialités diverses aidant à surpasser l'isolement et l'absence de confrontation des méthodes ${ }^{32}$.

À mon avis, la véritable brèche dans cet isolement fut l'exposition et le colloque Die Parler und der schöne Stil 1350-1400 tenus en 1978 à Cologne ${ }^{33}$. Anton Legner, directeur du Schnütgen-museum, à l'origine de ce projet, avait invité à participer à côté des spécialistes occidentaux leurs collègues des pays de l'Est. Il a réussi à produire le premier discours commun européen d'après-guerre sur ce thème.

Philippe Araguas. L'impact de la Seconde Guerre sur les monuments français a été mineur dans la mesure où peu d'entre eux ont été touchés par les destructions occasionnées par les bombardements: je n'ai pas connaissance de grands bouleversements des connaissances occasionnés par les restaurations (Carentan), ou par les reconstructions (Valognes!) dans les régions les plus atteintes... Des opérations de sauvegarde (je pense en particulier aux opérations de dépose et repose des vitraux) ont permis toutefois une prise de conscience "patrimoniale ». Elles sont à l'origine de travaux de fonds sur le vitrail lancés par Louis Grodecki.

Thomas Coomans. Après les ravages de la Première Guerre mondiale, le patrimoine belge paya de nouveau un lourd tribut pendant la Seconde Guerre mondiale (destructions de Nivelles et Tournai, bombardement d'Anvers, de Louvain, offensive des Ardennes, etc.). Toutefois, la restauration des monuments endommagés, considérée comme moins prioritaire, se fit beaucoup plus lentement et se prolongea, dans certains cas, jusque dans les années 1970. Comme en Allemagne, quoique moins systématiquement, les grandes églises dévastées firent l'objet de fouilles qui permirent d'en reconnaître les phases primitives. Une conséquence de la guerre froide fut la mise en place, en application de la Convention de La Haye (1954), d'un service interministériel pour la protection du patrimoine culturel en cas de conflit armé. Il lança un programme ambitieux de collecte d'une documentation scientifique utile à la restauration des monuments qui seraient détruits lors d'une nouvelle guerre - c'est alors que fut créé le service de photogrammétrie -, mais démontra l'absence d'une véritable politique patrimoniale concertée.

Dès le lendemain de la guerre, Raymond Marie Lemaire (1921-1997) entama une carrière brillante, d'abord dans l'historiographie de l'architecture gothique belge, puis, à un niveau international, pour la conservation du patrimoine. Auteur d'un doctorat en histoire de l'art sur l'origine du style gothique en Brabant ${ }^{34}$, il mit un point final à la théorie nationaliste flamande du "gothique thiois» de Stan Leurs dans les années 1930, et fixa la " géographie » des écoles régionales et subrégionales gothiques belges ${ }^{35}$. Celle-ci ne fut remise en question qu'après sa mort. Professeur d'histoire de l'architecture médiévale aux deux universités de Louvain de 1946 à 1986, Lemaire publia peu et s'orienta rapidement vers la restauration et l'expertise en conservation des monuments: coauteur de la Charte de Venise en 1964, puis cofondateur de l'ICOMOS dont il fut secrétaire général et président, il fonda en 1976 un post-graduat [cursus post-doctoral] international pour la conservation des 
monuments au Collège de l'Europe ${ }^{36}$. Lemaire fut également le promoteur, dès 1965, de l'inventaire systématique du patrimoine architectural en Belgique.

Peter Draper. Cela peut surprendre, mais ces événements n'ont eu que peu d'effets sur l'historiographie de l'architecture gothique dans les îles britanniques. Les principaux édifices ont été singulièrement épargnés (c'est sans doute que les pilotes de bombardiers les utilisaient comme points de repère), bien que certains d'entre eux aient été touchés au cours des raids Baedeker [nom donné à des raids de l'aviation allemande en 1942 contre des villes de peu d'importance stratégique mais distinguées dans les guides Baedeker]. Plusieurs cathédrales ont subi des dommages relativement légers, entre autres Canterbury, Exeter et Llandaff au pays de Galles (où on a profité de l'occasion pour "moderniser " l'intérieur du bâtiment, travail confié à George Pace et à Jacob Epstein pour les sculptures) mais les reconstructions nécessaires n'ont entrainé aucun accroissement notable des connaissances, aucun effet significatif dans le domaine historiographique. La cathédrale de Coventry, sérieusement endommagée, fut laissée en l'état pour servir de mémorial.

Reste que ces travaux ont pu soulever de nouvelles questions touchant les pratiques de la restauration et sur ce qui était souhaitable en la matière, qu'ils ont pu aussi attirer l'attention sur les changements apportés aux édifices gothiques dans les temps qui suivirent la Réforme, particulièrement aux XVIII ${ }^{e}$ et XIX ${ }^{e}$ siècles $^{37}$.

Pour répondre aux menaces que la guerre faisait peser sur les monuments, des archives nationales des bâtiments ont été créées, le National Monuments Record, et en 1941 le photographe Bill Brandt a été chargé de fixer l'image des édifices et du mobilier qui risquaient de disparaître. La guerre influa peut-être davantage sur l'étude des vitraux: on en avait décroché un grand nombre pour les mettre en lieu sûr et leur remise en place fut une bonne occasion de les restaurer et de mieux les connaître.

DANY SANDRon. Comment se traduisent dans l'historiographie les grandes mutations géopolitiques de notre époque (construction européenne, effondrement du bloc soviétique, unification de l'Allemagne)?

Bruno Klein. C'est avant tout la réunification qui a joué un rôle important pour la recherche en architecture gothique puisque de nombreux monuments, inaccessibles ou difficilement accessibles pendant des décennies, s'ouvraient à nouveau au contexte international. Il faudrait aussi ajouter l'amélioration et l'intensification des contacts avec les collègues des pays voisins de l'Est dont résultent nombre d'initiatives communes. À titre d'exemple, on peut mentionner le Dehio-Handbuch der Kunstdenkmäler pour la Silésie qui est le résultat d'une collaboration de chercheurs allemands et polonais, édité dans les deux langues ${ }^{38}$. Un problème se pose néanmoins dans le fait que l'histoire de l'art ouest-allemande s'est orientée pendant des décennies vers l'Ouest, ce qui est à la base d'une barrière linguistique importante dans le sens Ouest-Est, alors que les habitants des pays de l'Europe de l'Est pratiquent l'allemand.

Klára Benešovská. Les changements depuis 1989 sont énormes. Pour les historiens des anciens pays socialistes, l'ouverture des frontières a représenté une grande chance: voyager et connaître les œuvres d'art de visu, l'accès à la littérature, la participation à des colloques internationaux etc., la possibilité pour les étudiants de suivre un enseignement dans un autre pays, de surmonter les barrières linguistiques, 
d'acquérir l'expérience de milieux académiques divers. Quant à l'architecture gothique, les résultats de la recherche des dernières décennies étaient publiés dans la forme des grandes synthèses ainsi que des catalogues des expositions en Autriche, Hongrie, Pologne, Slovaquie, République tchèque ${ }^{39}$. Très rapidement on a rattrapé le retard en matière de technologie informatique. Naturellement la génération plus âgée est un peu moins à l'aise à cause de l'isolement imposé par les systèmes totalitaires, mais dans la plupart des cas les chercheurs se sont adaptés et réalisent ce qu'on ne pouvait faire avant 1989.

Par exemple les colloques internationaux sur l'art de l'époque de Jean l'Aveugle en $1996^{40}$, sur les tours des cathédrales en $2001^{41}$, sur les chapelles palatiales ${ }^{42}$, sur Peter Parler ${ }^{43}$ ou sur l'architecture autour $1300^{44}$ ont ouvert de nouvelles perspectives pour la collaboration internationale future. C'est comme si le combat pour la priorité de l'art « national » n'avait jamais eu lieu.

En Europe centrale et orientale, il faut apprécier les activités des collègues et des institutions allemandes pour ce qui est de l'organisation de diverses conférences, ainsi que la création de nouvelles institutions qui favorisent les études sur la culture de l'Ostmitteleuropa. Si la réflexion critique et ouverte de l'historiographie de l'art du $\mathrm{xx}^{\mathrm{e}}$ siècle se fait à un haut niveau, c'est avant tout grâce à l'historiographie allemande récente ${ }^{45}$

Je dirais que c'est là la meilleure réponse à notre passé commun.

Thomas Coomans. Tournée vers ses problèmes identitaires internes, la Belgique, pourtant considérée comme une "élève modèle de la construction européenne ", ne plaça pas son histoire de l'architecture dans une perspective internationale. Au contraire, une forte tendance au sous-régionalisme, fondé sur l'usage de matériaux locaux et de formes liées à ceux-ci, perdura tout au long de la seconde moitié du $\mathrm{xx}^{\mathrm{e}}$ siècle. Jusqu'à la fin des années quatre-vingts, les travaux des historiens de l'architecture médiévale tels que Luc Devliegher, Simon Brigode, Firmin De Smit, Frieda Van Tieghem, Michel De Waha ou Luc Genicot développèrent, certes de manière pionnière, une approche d'archéologie du bâti, mais furent incapables de renouveler l'approche de l'histoire de l'architecture gothique et de développer des contacts internationaux. Adelbrecht Van de Walle publia en 1971 une synthèse sur le gothique en Belgique ${ }^{46}$, qui est une compilation incohérente de sous-régionalismes, à partir de la division en écoles, groupes et sous-groupes établie par Raymond Lemaire. Il fallut attendre un quart de siècle avant que ne paraisse, en 1997, une nouvelle synthèse sur l'architecture gothique en Belgique ${ }^{47}$. Richement illustré, cet ouvrage de qualité inégale intègre les apports de restaurations récentes, mais ne parvient pas à s'affranchir entièrement des catégories identitaires géographiques ${ }^{48}$.

Les mutations géopolitiques internationales n'influencèrent donc pas l'historiographie de l'architecture gothique en Belgique qui, désormais, se décline, selon la région: Flandre, Wallonie et Bruxelles. Il faut noter que ces régions ainsi que les provinces financent désormais l'essentiel de la recherche et les restaurations, contrôlent les services archéologiques et patrimoniaux, et s'imposent de plus en plus comme éditeurs. Inutile de préciser que la qualité scientifique de la recherche et des publications, privilégiant le prestige patrimonial et la promotion touristique, en pâtit gravement. Ainsi, l'opération autour du gothique brabançon, lancée par la province de Brabant flamand en 2002, malgré l'organisation d'un colloque scientifique, ne put 
compter sur l'aide des autres provinces belges qui se divisent le territoire de l'ancien duché de Brabant - par exemple la province d'Anvers -, car leurs responsables politiques rejettent l'identité brabançonne. Seul le Brabant septentrional, province des Pays-Bas, réagit avec enthousiasme! Ce genre de situation n'est malheureusement pas propre à la Belgique et n'est guère de bon augure pour l'avenir.

Philippe Araguas. Mon expérience me donne une familiarité plus grande avec l'Europe méridionale qu'avec l'Europe du nord ou de l'est, je me contenterai donc de donner mon opinion sur ce seul domaine.

Je ne pense pas que la construction européenne ait joué le moindre rôle jusque dans les années 1990 dans le domaine de l'histoire de l'architecture gothique. Même si la magnifique entreprise qui a abouti en 1989 à l'exposition de Strasbourg Les bâtisseurs des cathédrales gothiques ${ }^{49}$ est contemporaine de l'acmé de ce qui reste aujourd'hui encore un projet politique au devenir incertain, je pense qu'elle doit beaucoup plus au réseau académique et intellectuel tissé par Roland Recht qu'à une quelconque manifestation de communautarisme européen. Les solidarités culturelles supranationales, notamment celle de l'Alsace avec l'Allemagne (que l'on peut qualifier de "naturelles" ou d'«ethniques" même si cela peut apparaître très politiquement incorrect), celle plus «historique» du monde «anglo-normand» auquel Jean Bony a magistralement et subtilement associé le monde franco-picard, ou celle de la France avec l'Espagne et l'Italie (liées à l'existence d'institutions comme l'École française de Rome ou la Casa Velazquez) sont indépendantes et souvent antérieures au projet même de communauté européenne. Est-ce à dire que les frontières sont infranchissables? Certes non: on note, incontestablement, dans le cours des cinquante dernières années, un apport très considérable des historiens allemands à la connaissance du gothique français (Sauerländer, Kimpel, Suckale, Freigang...) mais il ne m'apparaît pas spécifiquement lié à la construction européenne dans le sens politique et réducteur que l'on donne à ce concept. Sans doute en sera-til très différent dans les années à venir, quand les possibilités d'échanges et l'ouverture des postes de chercheurs et d'enseignants à tous les ressortissants de la communauté auront opéré un réel brassage comme cela commence à se faire.

Pour ce qui est de l'Espagne, que je connais bien, et de l'Italie, que je connais moins bien, il me semble que les changements proviennent davantage du corollaire de la construction européenne que constitue l'affirmation des spécificités régionales, que de l'Union Européenne. Cela est peu sensible en France ou, dans le domaine de l'architecture gothique, le vieux concept d'écoles régionales apparaît fortement battu en brèche et n'a pas bénéficié de la balbutiante régionalisation mise en œuvre avec détermination mais conduite selon un train de sénateur... En Espagne, l'historiographie du gothique, dominée longtemps par une pensée assez clairement nationaliste ou du moins castillanocentriste étayée par le livre fondateur d'Élie Lambert L'art gothique en Espagne (1931) ${ }^{50}$, se tourna vers une approche plus régionaliste avec la thèse de Marcel Durliat sur l'art dans le Royaume de Majorque publié en $1962^{51}$ qui établissait un pont entre la France du Général de Gaulle et l'Espagne du Général Franco dans le plus superbe dédain des considérations nationales. La réalité de courants régionalistes puissants, perçue de longue date par des historiens de l'architecture comme Lampérez ${ }^{52}$ fut, à partir des années 1980 , soulignée avec virulence par de multiples études financées ou publiées par les autonomies, les collectivités régionales et locales et les entités bancaires à forte 
implantation locale comme les caisses d'épargne. Ainsi, la richesse documentaire accumulée, selon une méthodologie qui doit beaucoup au modèle académique français (le Centre d'études médiévales de Poitiers a joué auprès d'autres institutions de recherches françaises un rôle important dans la diffusion de ce modèle durant les années du franquisme), y est encore accrue par la richesse incomparable des fonds médiévaux. Ce phénomène de multiplication des études régionales est, me semble-til, identique, quoique sans doute plus ancien en Italie, et l'on ne peut que regretter que la force d'inertie du jacobinisme français interdise que l'on puisse faire le même constat pour la France. Certes, dans ce domaine, on ne peut que se réjouir de l'entreprise éditoriale de Picard lancée par Anne Prache, relayée ici et là par des éditeurs régionaux, qui offre une collection d'ouvrages un peu comparables pour le monde gothique à ce que constitua dans les années 1960-1970 la collection Zodiaque pour le monde roman. Il n'échappera à personne cependant que ces ouvrages ne constituent le plus souvent que de commodes recueils de monographies plus ou moins habilement cousues ensemble par des synthèses limitées par le parti même de la monographie régionale.

Francesca Español. Sur le plan historiographique, la nouvelle situation européenne n'a guère eu d'écho en Espagne. Bien qu'il faille être critiques en évaluant les questionnements qui régissent la communauté des historiens de l'art, bon nombre d'entre eux, attentifs et informés, connaissent, et depuis longtemps, les chemins par lesquels transite notre discipline et ses instruments méthodologiques au-delà des Pyrénées. Ce qu'il faut espérer, c'est que cette situation s'inverse car, dans de nombreux cas, la connaissance qu'ont les historiens espagnols de leurs collègues étrangers est plus importante que celle que ces derniers ont de nous.

Il se peut que le seul domaine pour lequel on ait noté une incidence (bien que je ne sois pas très optimiste sur la question) s'inscrive dans le cadre des projets internationaux financés par l'Union européenne, dont a bénéficié, par exemple, la cathédrale de Gérone.

À mon avis, ce sont plutôt les nouvelles technologies qui ont supposé un changement radical dans les habitudes des historiens, bien que cela soit moins imputable à la nouvelle situation géopolitique qu'aux nouvelles possibilités offertes par l'époque actuelle. La facilité d'accès à l'information à tous les niveaux grâce à l'internet est révolutionnaire. De la maison, de notre bureau à l'université ou au centre de recherche, nous touchons les musées, les bibliothèques, médiathèques et centres d'archives, le matériel digitalisé etc. Je souhaite également souligner qu'il est précieux d'avoir accès aux nouvelles concernant les congrès sur la recherche, les expositions etc.

Peter Draper. C'est une question intéressante, mais on a du mal à repérer les courants de l'historiographie contemporaine qui pourraient se rattacher avec certitude aux grands événements géopolitiques de la seconde moitié du $\mathrm{xx}^{\mathrm{e}}$ siècle. Sans doute l'intérêt pour l'architecture continentale s'est-il accru, encouragé par une plus grande facilité de déplacement, surtout vers l'Europe de l'Est. Les récents travaux de Paul Crossley sur l'Europe centrale témoignent d'un élargissement des perspectives traditionnelles des études ins ulaires, mais ses recherches ont débuté en Pologne au cours des années 1960, elles ne sont pas la conséquence des événements dont nous parlons. 
DANY SANDRON. À l'heure de la mondialisation, peut-on estimer la part prise dans le domaine géographique qui vous concerne par les chercheurs étrangers non ressortissants du pays, qu'ils soient originaires d'autres pays d'Europe, des États-Unis, voire d'Asie? Est-ce un facteur de renouvellement des approches et des problématiques?

Thomas Coomans. Si la peinture, l'enluminure, les retables sculptés, les tapisseries et la dinanderie du "siècle des primitifs flamands " dans les anciens Pays-Bas font depuis longtemps l'objet d'études, de publications et d'expositions au plus haut niveau international, il en va tout autrement de l'architecture. Le nombre de chercheurs non belges ou hollandais qui se risquent sur le terrain de l'architecture gothique dans cette zone géographique est extrêmement limité.

Je vois plusieurs raisons principales à ce paradoxe. Tous les rapports et les publications archéologiques et architecturales, y compris des restaurations, concernant les Pays-Bas et la Flandre sont en néerlandais, exceptionnellement en français, jamais en anglais. Mais cet obstacle linguistique n'explique pas tout. Datant surtout de la seconde moitié du XIV ${ }^{\mathrm{e}}$ au début du XVI ${ }^{\mathrm{e}}$ siècle, le patrimoine gothique des anciens Pays-Bas, malgré ses chefs-d'œuvre inégalés, attire beaucoup moins que le gothique des XII $^{\mathrm{e}}$ et XIII ${ }^{\mathrm{e}}$ siècles qui, jusqu'il n'y a pas tellement longtemps, capturait toute l'attention des chercheurs. En outre, il est clair que depuis les missions allemandes dirigées par Paul Clemen pendant la Première Guerre mondiale, les historiens de l'architecture allemands ont longtemps jugé opportun d'éviter la Belgique. Par ailleurs, comme je l'ai déjà dit, les titulaires de chaires d'histoire de l'architecture en Belgique, pourtant médiévistes, se replièrent sur leur sous-région et ne développèrent pas des réseaux internationaux. Enfin, les études historiques, fort développées pour le bas Moyen Âge, s'intéressent également aux phénomènes artistiques, à la culture de l'image (dévotions, représentation), aux paysages culturels etc., mais guère à l'architecture. Lorsqu'il s'agit d'approche archéologique et architecturale, la multidisciplinarité avec les historiens semble particulièrement difficile.

Je voudrais ici souligner l'ouverture d'esprit de certains historiens contemporanéistes, moins enfermés dans leurs a priori méthodologiques que leurs collègues médiévistes, qui reconnaissent depuis longtemps à l'architecture le rôle essentiel qui lui revient dans la société des $\mathrm{XIX}^{\mathrm{e}}$ et $\mathrm{XX}^{\mathrm{e}}$ siècles. À cet égard, la communauté de recherche internationale "Architecture, conceptions du monde et identités culturelles en Europe occidentale 1815-1945», mise sur pied en 2002 par Jan De Maeyer, professeur à l'université flamande de Louvain, mérite une mention particulière pour sa pluridisciplinarité. Il existe également des historiens de l'architecture gothique qui ne méprisent pas l'architecture néo-gothique, les restaurations historicistes et les questions liées à la réception du Moyen Âge à l'époque industrielle et à sa diffusion dans les colonies. Ainsi se développent des regards croisés que je considère comme féconds et parfois même indispensables. Ils facilitent d'ailleurs la compréhension de l'historiographie de l'architecture gothique aux XIX et $\mathrm{XX}^{\mathrm{e}}$ siècles.

Le programme « Kunst und Region/Art and Region » dirigé de 1995 à 2000 par Aart Mekking, professeur à l'Université de Leyde, a amorcé un autre changement. Ce programme de rencontres et de visites, auquel participèrent une cinquantaine de jeunes chercheurs hollandais, allemands et belges, promut une nouvelle approche de l'architecture médiévale, au-delà des paysages culturels nationaux (Kunstlandschaft), 
en favorisant l'approche iconologique de l'architecture et la recherche de la signification culturelle et historique des bâtiments dans leurs contextes. Adepte des théories développées par Günther Bandmann à partir de 1951, Aart Mekking refusait l'héritage de l'historiographie nationaliste et développa une vision internationale et même transculturelle. Ce réseau ne donna lieu qu'à une publication ${ }^{53}$, mais les idées et la méthode qui s'y développèrent nourrirent des doctorats et sont présentes dans de nombreuses publications. Les deux années postdoctorales passées chez Mekking à Leyde m'ont ouvert à une histoire de l'architecture médiévale, d'origine allemande, inconnue du monde académique belge.

Ceci ne signifie pas que les chercheurs belges ne sortent pas de leurs frontières, mais ceux qui bougent restent dans des réseaux très spécialisés, liés à la restauration, à l'archéologie et à l'archéologie du bâti (comme le « Arbeitskreis für Hausforschung ", la dendrochronologie et les autres disciplines archéométriques, la glyptographie et la taille de la pierre, etc.), ou à des arts appliqués à l'architecture (comme les vitraux et les peintures murales). Il n'y a pas, disons plutôt qu'il n'y a pas encore de réseau international structuré pour l'architecture gothique comme il en existe pour la Renaissance, certains ordres religieux, la castellologie, les vitraux, etc. L'appel est lancé.

Philippe Araguas. C'est effectivement le regard extérieur porté par des chercheurs étrangers qui, à mon sens, permet d'abolir les limites d'une recherche contrainte par sa rigueur encore fortement empreinte du positivisme de l'École des chartes ancienne manière (car cette vénérable institution a su, elle aussi, évoluer). Les travaux magistraux de Jean Bony, ceux articulant scrupuleusement analyse monographique et vision de synthèse de Kimpel et Suckale pour ne citer qu'eux, ont constitué un apport considérable à la compréhension de l'architecture gothique en s'appuyant sur les données de l'archéologie monumentale accumulées pendant plus d'un siècle d'études érudites. Mais c'est d'autres chercheurs et d'autres regards jetés sur cette architecture que provient le renouvellement d'une science quelque peu engoncée à mon avis dans le ronronnement douillet d'une archéologie monumentale bien réglée. Je laisse délibérément de côté les apports attendus de l'archéologie du bâti qui n'est à mes yeux que l'avatar "high-tech» de l'archéologie monumentale, mais je pense nécessaire de mentionner une approche «low tech» de l'archéologie du bâti qui n'a pas me semble-t-il reçue en France l'accueil qu'elle eût mérité; je veux parler des travaux menés par John James, architecte et historien de l'architecture australien dont les études sur Chartres et sur les églises de l'île-de-France constituent une source considérable de profit pour la réflexion sur l'architecture gothique ${ }^{54}$.

Mais au-delà de cette approche somme toute très archéologique de l'architecture gothique, celles plus purement spirituelles ou intellectuelles du phénomène, de Otto Von Simpson ou Erwin Panofsky, ont modifié le courant de l'historiographie gothique et suscité, notamment auprès des historiens anglo-saxons et américains, un élan de spéculations parfois échevelées. La synthèse de ces approches, ancrée dans la tradition positiviste de l'historiographie française, très attentive aux aspects techniques, statiques, stylistiques de la construction mais stimulée par une histoire de l'architecture plus spéculative, constitue la spécificité des apports de Stephen Murray et de son école qui ont largement contribué à rajeunir les travaux dans ce domaine. On ne peut passer non plus sous silence la grande bouffée d'oxygène que les analyses spatiales et les réflexions sur les tracés proposées par Robert Branner a 
constitué pour les historiens de ma génération. Enfin, pour en rester dans les apports de l'Amérique du Nord, on se doit de mentionner, même si elle apparait inaboutie, l'étude pionnière de Roland Sanfaçon sur le gothique flamboyant ${ }^{55}$ dont les prolongements, par le biais d'études menées par certains de ses disciples, rejoignent heureusement celles menées par des jeunes chercheurs de tradition plus strictement chartiste, sur le gothique tardif.

Francesca Español. L'art espagnol intéresse depuis le $\mathrm{XIX}^{\mathrm{e}}$ siècle les historiens étrangers, et dans le domaine de l'architecture gothique se détachent des pionniers tels que l'Anglais George Edmund Street, l'Allemand Augusto L. Mayer et les Français Élie Lambert et Pierre Lavedan. Marcel Durliat, plus récemment, a consolidé la tradition hispaniste française qui perdure actuellement grâce à Philippe Araguas ou Jean Passini dans le domaine de l'urbanisme. Parmi les Allemands, il convient de signaler Christian Freigang avec son ouvrage sur l'architecture gothique espagnole de 199956, ou la biographie consacrée à Jaume Fabre, architecte des cathédrales de Narbonne et Gérone en 1989, de même que la monographie sur la cathédrale de Burgos de Henrik Karge ${ }^{57}$. L'étude de John D. Hoag consacrée à Rodrigo Gil de

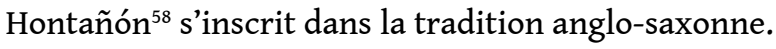

Dans cet ensemble de travaux, même le plus ancien, Gothic Architecture in Spain de Street $(1865)^{59}$, a été tardivement connu par les Espagnols, car il a fallu en attendre assez longtemps la traduction (1926). Il convient donc d'en écarter l'éventuelle influence méthodologique. En ce qui concerne les travaux d'Élie Lambert et Pierre Lavedan, ils sont apparus quand l'art espagnol médiéval, particulièrement l'architecture, avait déjà été abordé par des historiens autochtones de l'importance de Josep Puig i Cadafalch ou Manuel Gómez Moreno - bien qu'il faille reconnaître que dans les premières années $\mathrm{du} \mathrm{xx}^{\mathrm{e}}$ siècle, l'art roman a pris le pas sur l'art gothique et que les deux spécialistes espagnols, puis à leur suite Lampérez et Torres Balbás, ont davantage consacré leur attention aux constructions des $\mathrm{XI}^{\mathrm{e}}, \mathrm{xII}^{\mathrm{e}}$ et $\mathrm{XIII}{ }^{\mathrm{e}}$ siècles qu'à celles du bas Moyen Âge. Les Espagnols analysaient les structures, et ils comparaient aussi les relevés et ont eu recours aux données documentaires pour comprendre le développement de l'architecture dans le temps et l'origine des solutions architecturales mises en œuvre dans les édifices. Le problème ne tient souvent pas tant au manque d'intérêt supposé des historiens espagnols pour leurs monuments qu'à la fréquente méconnaissance de l'historiographie internationale des travaux réalisés et publiés en Espagne. Cette situation paradoxale se produit dans une moindre mesure dans le domaine historique ou dans celui de la littérature médiévale où les échanges entre spécialistes sont beaucoup plus fluides. J'insistais auparavant sur la valeur des nouvelles technologies. Dans notre discipline, l'accès aux revues spécialisées internationales est une chose commune à nombre d'entre nous et c'est de là que provient le renouvellement méthodologique que reflètent les recherches les plus récentes. La difficulté d'une consultation inverse, justifie, même si elle n'excuse pas, le silence de la bibliographie étrangère sur des travaux espagnols remarquables et de référence.

Peter Draper. Les années 1930 ont été marquées par l'influence intellectuelle de la diaspora. Nikolaus Pevsner prédomine après-guerre avec la publication de son Outline of European Architecture (Londres, 1943), son travail de direction éditoriale pour la Pelican History of Art et surtout ses études consacrées aux monuments médiévaux dans la collection Buildings of England ${ }^{60}$. Pevsner a élargi le cadre de nos références, en 
établissant des parallèles avec l'architecture continentale, en insistant sur l'importance d'une meilleure connaissance du Gothic Revival et des restaurations effectuées au XIX ${ }^{e}$ siècle. L'ampleur de son influence a été analysée sous ses divers aspects dans un ouvrage collectif récent, avec une magistrale introduction de Paul Crossley ${ }^{61}$.

D'ordinaire, l'architecture médiévale des îles britanniques n'a guère mobilisé les chercheurs hors du Royaume-Uni, si l'on excepte ce qu'il est convenu d'appeler le Decorated Style du XIv ${ }^{\mathrm{e}}$ siècle avec les études de Bock et de Bony ${ }^{62}$. Dans leur conception, ces deux livres présentent un contraste fascinant, Bock représentant la critique stylistique allemande et Bony une tradition française d'analyse stylistique affinée à son plus haut niveau. On peut prendre une mesure précise des changements d'intérêts, d'approches et d'interprétations qui se dessinent au cours des décennies suivantes, en comparant ces deux ouvrages avec celui de Nicola Cold-stream ${ }^{63}$, un livre qui se distingue notamment par sa prise en compte de l'architecture séculière et d'un éventail d'objets bien plus large.

D'autres chercheurs allemands ont étudié l'architecture gothique des îles britanniques ${ }^{64}$ : ces ouvrages rejoignaient parfois les préoccupations des Britanniques mais ils étaient publiés en allemand, difficilement accessibles au Royaume-Uni et ils n'ont pas trouvé l'audience qu'ils méritaient.

Les chercheurs américains (Peter Fergusson, Larry Hoey, Malcolm Thurlby) ont donné des contributions importantes, mais en demeurant dans le sillage de la tradition académique anglaise et sans proposer d'approche vraiment nouvelle.

Les changements de perspective dans l'étude de l'architecture gothique des îles britanniques, si manifestes à partir des années 1980, ne sont pas le fait des spécialistes étrangers du domaine anglais: ils procèdent plutôt d'une plus grande attention aux nouvelles approches que les autres pays ont appliquées à leurs propres architectures. Un phénomène qui s'est affirmé dans le cadre des colloques internationaux (celui de Poitiers, par exemple), favorisé par la publication d'index bibliographiques internationaux et, plus récemment, par les ressources auxquelles internet donne accès.

Bruno Klein. Apparemment, il n'existe pas de mondialisation des recherches sur l'architecture gothique qui aille au-delà de ce que nous connaissons déjà (par exemple la recherche sur le gothique aux États-Unis). Le phénomène de la mondialisation, essentiellement porté sur l'économie et sur la culture du quotidien, n'intervient que de façon marginale dans les recherches en histoire.

Cependant, l'échange déjà mentionné avec des collègues de l'Europe de l'Est est un autre facteur qui s'entend comme un résultat des bouleversements politiques de la fin des années 1980 aux années 1990. Grâce à cet échange, les monuments gothiques de ces pays ont été réintroduits dans le centre d'intérêt des chercheurs, ce qui a pour effet un réajustement des perspectives de la recherche. L'intérêt devrait particulièrement porter sur les questions généralement liées au transfert culturel historique.

Pour l'Europe de l'Ouest, on peut constater que, depuis ces dernières années, l'architecture gothique de l'Espagne, du Portugal, et même celle des États d'Amérique latine, occupe une place importante dans les recherches en Allemagne. Sur ce point, 
la Carl-Justi-Vereinigung zur Förderung der kunstwissenschaftlichen Zusammenarbeit mit Spanien und Portugal (Association Carl Justi pour le soutien de la collaboration en histoire de l'art avec l'Espagne et le Portugal) a rendu de grands services.

Klára Benešovská. L'ouverture de l'Europe centrale et orientale au monde occidental et ses conséquences pour notre discipline peut revêtir là aussi des aspects divers. Les thèmes traditionnels des historiographies nationales des pays de cette région se sont internationalisés avec l'acceptation de la pluralité des méthodes. Les sujets régionaux se sont ouverts à tous, que les chercheurs aient étudié aux États-Unis, en Australie ou en Lituanie. La seule exigence est le contexte de l'œuvre, qui est lié au moment historique de sa production et à la culture du pays où elle a été créée ou bien pour lequel elle a été commandée. On ne devrait pas non plus ignorer les sources locales, la littérature spécialisée régionale écrite dans la langue du pays en question et les realia locales. Ce n'est pas toujours le réflexe dans le cas des travaux de synthèse ou des catalogues de grandes expositions.

La confrontation d'avis différents sur des thèmes spécifiques, la possibilité de débattre, l'amélioration des contacts entre spécialistes, aussi grâce à l'internet, sont des avantages indiscutables. En ce sens, la recherche sur l'architecture gothique peut aussi profiter de plusieurs méthodes suivant le sujet. L'avenir appartient à la collaboration interdisciplinaire et internationale.

Mais il y a aussi le revers de la médaille: dans le domaine de la protection du patrimoine national on se trouve confronté à de vrais dangers. Les anciens pays socialistes se sont ouverts aux investissements internationaux, aux sociétés multinationales. La pression pour la construction et la reconstruction dans les centres historiques, mais aussi dans le paysage périurbain, est énorme. La protection des monuments et des sites n'est pas encore bien définie d'un point de vue législatif, sans parler des menaces de corruption. De plus, le postmodernisme en architecture a mis l'accent sur l'imitation des modèles historiques. Les nouvelles constructions grandioses qui ne respectent pas la structure et le panorama des villes ne devraient pas laisser sans réaction les historiens de l'art. La protection du patrimoine reste donc encore partie intégrante de notre discipline - la tradition de l'école de Vienne a toujours cours. Mon expérience à Prague me donne espoir - ces dernières années, ce sont les étudiants en histoire de l'art qui jouent le rôle des gardiens non-officiels du patrimoine.

\section{NOTES DE FIN}

1. Marvin Trachtenberg, "Gothic/Italian 'Gothic': Toward a Redefinition », dans Journal of the Society of Architectural Historians, mars 1991, p. 22-37.

2. Caroline Bruzelius, The Stones of Naples: Church Building in the Angevin Italy, 1266-1343, New Haven/ Londres, 2004 ; éd. italienne : Le pietre di Napoli : l'architettura religiosa nell'Italia angioina, 1266-1343, Rome, 2005. 
3. Christian Freigang, "Imitare Ecclesias nobiles » Die Kathedralen von Narbonne, Toulouse und Rodez und die nordfranzösische Rayonnantgotik im Languedoc, Worms, 1992.

4. Henrik Karge, La Catedral de Burgos y la Arquitectura del siglo XIII en Francia y España, Junta de Castille-León, 1995 ; éd. originale : Die Kathedrale von Burgos und die spanische Architektur des 13. Jahrhunderts, Berlin, 1987.

5. Lindy Grant, Architecture and Society in Normandy, 1120-1270, éd. originale, New Haven/Londres, 2005.

6. Christopher Wordsworth, Ceremonies and Processions of the Cathedral Church of Salisbury, 1901, et W. St John Hope, «Quire screens in English Churches with special references to the 12th-century screen formerly in the Cathedral Church of Ely ", dans Archeologia LXVIII (1917), p. 43-110.

7. Thomas Cocke, Peter Kidson, Salisbury Cathedral: Perspectives on the Architectural History, Londres, 1993.

8. Sarah Brown, Sumptuous and richly adorn'd : the decoration of Salisbury Cathedral, Londres, 1999.

9. Sarah Pearson, The Medieval Houses of Kent, Londres, 1994.

10. Virginia Raguin, Kathryn Brush, Peter Draper éd., Artistic integration in Gothic Buildings, Toronto, 1995.

11. Eric Fernie, Paul Crossley éd., Medieval Architecture and its Intellectual Context : Studies in Honour of Peter Kidson, Londres, 1990.

12. À ce propos, on peut aussi consulter les essais recueillis dans G. Clarke, P. Crossley éd., Architecture and Language: Constructing Identity in European Architecture c.1000-c.1650, Cambridge, 2000.

13. A. G. B. Schayes, Mémoire sur l'architecture ogivale en Belgique, (Mémoires couronnés par l'Académie royale des Sciences et Belles-Lettres de Bruxelles, 14/2), Bruxelles, 1839, p. 1-194.

14. H. Janse, R. Meischke, J.H. van Mosselveld, F. Van Tyghem, Keldermans. Een architectonisch netwerk in de Nederlanden, La Haye/Bergen op Zoom, 1987.

15. Dieter Kimpel, Robert Suckale, Die gotische Architektur in Frankreich 1130-1270, Munich, 1985 (trad. fr. : L'architecture gothique en france, 1130-1270, Paris, 1990).

16. Willibald Sauerländer, Gotische Skulptur in Frankreich, 1140-1270, Munich, 1970. (trad. fr. : La sculpture gothique en France, 1140-1270, Paris, 1972).

17. Die Parler und der Schöne Stil 1350-1400. Europäische Kunst unter den Luxemburgern ", (cat. exp., Cologne, 1978), Cologne, 1978 ; Prague. The Crown of Bohemia, Jiří Fajt éd., (cat. expo., New York, 2005), New York/Prague, 2006.

18. Surtout dans son livre Deutsche Kirchenbaukunst der Gotik, Cologne, 1985 ; réédité en 1994.

19. Voir son article de 1873: Moriz Thansing "Die Stellung der Kunst-geschichte als Wissenschaft », repris dans Wiener Jahrbuch für Kunstgeschichte, XXXVI, 1983, p. 140-150.

20. Ján Bakoš, « From Universalism to Nationalism. Transformation of Vienna School in Central Europe ", dans Robert Born, Alena Janatková, Adam S. Labuda éd., Die Kunsthistoriographien in Ostmitteleuropa und der nationale Diskurs, Berlin 2004, p. 79-101.

21. Pour l'analyse de l'historiographie de l'architecture gothique dans le cadre de l'école de Vienne, voir Roland Recht, «La première et la seconde école de Vienne » dans Le croire et le voir. L'art des cathédrales ( $x i^{e}{ }^{e}-x v^{e}$ siècle), Paris 1999, p. 49-62, avec la bibliographie de base.

22. Voir Georg Dehio, "Neue Strömungen in der Denkmalpflege", dans Mitteilungen der k.k.Zentral-Kommission für Erforschung und Erhaltung der Kunst- und historischen Denkmäler, Vienne, 1905, p. 85-104.

23. Joseph Neuwirth, Geschichte der christlichen Kunst in Böhmen bis zum Aussterben der Přemysliden, Prague, 1888 ; id., Geschichte der bildenden Kunst in Böhmen vom Tode Wenzels III. bis zu den Hussitenkriege, Prague, 1893 ; id., Geschichte der deutschen Kunst und des deutschen Kunstgewerbes in den Sudetenländern bis zum Ausgang des 19. Jahrhunderts, Augsburg, 1926.

24. Pour Birnbaum, l'évolution formelle de l'art se transmet d'un pays à l'autre pour aboutir au plus haut degré de la qualité et de la créativité dans les circonstances historiques spécifiques de 
tel ou tel pays (ce qu'il appelait la «loi de la transgression») : Vojtěch Birnbaum, Doplněk $h$ v ývojové souvislosti v umění, Prague, 1987. Voir en particulier la conclusion d'Ivo Hlobil, p. 379-411). 25. Karl Maria Swoboda, «Zum deutschen Anteil an der Kunst der Sudetenländer », dans Beiträge zur Geschichte der Kunst in Sudetenraum I., Brünn, 1938 ; id., Peter Parler. Der Baukünstler und Bildhauer, Vienne, 1940 ; Otto Kletzl, Peter Parler, der Dombaumeister von Prag, Leipzig, 1940 ; id., Die deutsche Kunst in Böhmen und Mähren, Prague, 1941; Erich Bachmann a publié les résultats de ses recherches sur l'architecture gothique dans K. M. Swoboda éd., Gotik in Böhmen, Munich, 1969.

26. Wolfgang Schenkluhn, Die Architektur der Bettelorden. Die Baukunst der Domenikaner und Franziskaner in Europa, Darmastadt, 2000.

27. Matthias Untermann, Forma Ordinis - die mittelalterliche Baukunst der Zisterzienser, Munich, 2001 ; id., Architektur im Mittelalter, Darmstadt, 2006.

28. Václav Mencl, Česká gotická architektura doby lucemburské, Prague, 1948; traduction anglaise :Czech Architecture of the Luxembourg Era, Prague, 1955.

29. Les débuts de Václav Mencl comme historien de l'architecture médiévale sont liés à la Slovaquie. Il y est venu de Prague pour travailler dans le domaine de la protection des monuments puis comme professeur à l'université. Il y a publié des ouvrages fondamentaux sur l'architecture médiévale en Slovaquie : Bratislava, stavební obraz města a hradu, Prague, 1936 ; Stř edověká architektura na Slovensku, Prague, 1937 ; Středověká města na Slovensku I, II, Prague, 1937/ Bratislava, 1938.

30. Ce n'est bien sûr qu'une simplification de sa théorie très élaborée, mais limitée par ce dualisme dû à la tradition des méthodes évolutionnistes et de la Gestaltpsychologie.

31. Rudolf Chadraba éd., Dějiny českého výtvarného umění I/1,2, Prague, 1984 (les chapitres sur l'architecture gothique sont de Dobroslav Libal et Jirina Horejsi) ; Jaromir Homolka,Vaclav Mencl et al., Pozdněgotické umění v Čechách, Prague, 1978 (les chapitres sur l'architecture gothique flamboyante sont de Vaclav Mencl).

32. Un bon exemple est donné par la femme de V. Mencl, Dobroslava Menclova, qui, en dehors des institutions officielles mais soutenue moralement par des amis en France, a publié une synthèse fondamentale sur les châteaux médiévaux en Bohême (České hrady I-II, Prague, 1972).

33. Die Parler und der Schöne Stil 1350-1400, cité n. 17.

34. Raymond Lemaire, Les origines du style gothique en Brabant. Deuxième partie : la formation du style gothique brabançon, 1, Les églises de l'ancien quartier de Louvain, Anvers, 1949.

35. Raymond Lemaire, «L'architecture gothique », dans Paul Fierens éd., L'art en Belgique, 4 e éd., vol. 1, Bruxelles, 1957, p. 67-100.

36. L'actuel Centre Raymond Lemaire pour la Conservation, à la Katholieke Universiteit Leuven. 37. Un ouvrage de Gerald Cobb porte sur ce sujet: English Cathedrals. The Forgotten Centuries. Restoration and Change from 1530 to the Present Day, Londres, 1980.

38. Slawomir Brzezicki, Ernst Badstübner, Dehio-Handbuch der Kunstdenkmäler in Polen: Schlesien, Munich/Berlin/Varsovie, 2005.

39. Árpád Mikó, Imre Takács éd., Pannonia regia, Müvészet a Dunántúlon 1000-1541, Budapest, 1994 ; Jiří Kuthan, Česká architektura v době posledních Přemyslovců. Města-hrady-kláštery-kostely, Vimperk 1994. Voir aussi l'ensemble des textes de J. Kuthan sur l'architecture gothique cistercienne : Aleš Mudra ed., Gloria Sancti Ordinis Cisterciensis, Prague, 2005, et Teresa Mroczko, Marian Arszyński, Andrzej Wlodarek éd., Architektura gotycka w Polsce, [L'Architecture gothique en Pologne], 4 vol., Varsovie, 1995 ; Günter Brucher éd., Geschichte der bildenden Kunst in Österreich. Gotik, Vienne, 2000 ; Dušan Buran éd., Gotika. Dejiny slovenského výtvarného umenia, Bratislava, 2003. Les essais sur l'architecture gothique ont trouvé leur place aussi dans les catalogues des dernières grandes expositions sur l'art des Luxembourg en 2005-2006 : Jiří Fajt éd., Charles IV, cité n. 17 ; Imre Takács éd., Sigismundus rex et imperator, (cat. exp., Budapest/Luxembourg, 2006), Budapest/ Luxembourg, 2006. 
40. Klára Benešovská éd., King John of Luxemburg (1296-1346) and the Art of his Era (Proceedings of the International Conference, Prague, september 16-20, 1996), Prague, 1998.

41. "Les tours des cathédrales. Leur constructions et leur fonction », dans Umění XLIX/2001, p. 195-320.

42. Jiří Fajt éd., Court Chapels of the High and Late Middle Ages and their Artistic Decoration. Proceedings from the International Symposium, Couvent St Agnes, Prague 23-25 sept. 1998, Prague, 2004.

43. Parlerbauten. Architektur, Skulptur, Restaurierung, (colloque, Stuttgart, 17-19 janvier 2001), Stuttgart, 2004.

44. Alexandra Gajewski, Zoë Opacic éd., The Year 1300 and the Creation of a New European Architecture (Architectura Medii Aevi, 1), Turnhout, 2007.

45. Voir Robert Born, Alena Janatková, Adam S. Labuda éd., Die Kunsthistoriographien in Ostmitteleuropa und der nationale Diskurs, Berlin 2004, avec en particulier l'article de Thomas DaCosta Kaufmann, qui éclaire très bien la situation (" Die Geschichte der Kunst Ostmitteleuropas als Herausforderung für die Historiographie der Kunst Europas », p. 51-66).

46. Adelbrecht Van de Walle, Belgique gothique : architecture, art monumental, Bruxelles, 1971.

47. Marjan Buyle, Thomas Coomans, Jan Esther et Luc Genicot, Architecture gothique en Belgique, Bruxelles, 1997.

48. Celles-ci sont abandonnées dans Thomas Coomans, "L'architecture », dans Christian Heck éd., L'art flamand et hollandais. Le siècle des Primitifs 1380-1520, Paris, 2003, p. 17-121.

49. Roland Recht, Les bâtisseurs des cathédrales gothiques, (cat. exp., Strasbourg, Ancienne douane, 1989), Paris/Strasbourg, 1989.

50. Élie Lambert L'art gothique en Espagne aux xii et xiii ${ }^{e}$ siècles, Paris, 1931.

51. Marcel Durliat, L'art dans le Royaume de Majorque. Les débuts de l'art gothique en Roussillon, en Cerdagne et aux Baléares, Paris, 1962.

52. Vincente Lampérez, Historia de la arquitectura cristiana española en la edad media según el estudio de los elementos y los monumentos, 2 vol., Madrid, 1908-1909.

53. Uta Maria Bräuer, Emanuel Klinkenberg, Jeroen Westerman éd., Kunst \& Region. Architektur und Kunst im Mittelalter. Beiträge einer Forschungsgruppe / Art \& Region. Architecture and Art in the Middle Ages. Contributions of a Research Group (Clavis Kunsthistorische Monografieën, 20), Utrecht, 2005.

54. Il me semble à cet égard devoir souligner comme particulièrement troublant le fait que l'accueil assez mitigé réservé à ce chercheur dans le milieu universitaire ait pu être largement compensé par l'investissement de la société archéologique d'Eure-et-Loire dans la publication des trois volumes de son étude sur la cathédrale de Chartres.

55. Roland Sanfaçon, L'architecture flamboyante en France, Québec, 1971.

56. Christian Freigang éd., Gotische Architektur in Spanien/Arquitectura gotica en Espana (Ars Iberica, 4, Kunsthistoriche Studien der Carl justi-Vereinigung), Francfort-sur-le-Main, 1999.

57. Henrik Karge, La catedral de Burgos..., cité n. 4.

58. John D. Hoag, Rodrigo Gil de Hontañón - gótico y renacimiento en la arquitectura española del siglo XVI, Madrid, 1985.

59. George Edmund Street, Some account of gothic architecture in Spain, Londres, 1865. (trad. esp. : Madrid, 1926).

60. Celles qui concernent les cathédrales ont été remises à jour et republiées par Priscilla Metcalf, The Cathedrals of England, Harmondsworth, 1985.

61. Paul Crossley éd., Reassessing Nikolaus Pevsner, Londres, 2004.

62. Henning Bock, Der Decorated Style. Untersuchun-gen zur Englischen Kathedralarchitektur der ersten Hälfte des 14 Jahrhunderts, Heidelberg, 1962, et Jean Bony, The English Decorated Style. Gothic Architecture Transformed 1250-1350, Oxford, 1979.

63. Nicola Coldstream, The Decorated Style. Architecture and Ornament 1240-1360, Londres, 1994. 
64. Günther Kowa, Architektur der Englischen Gotik, Cologne, 1990 ; Ute Engel, Die Kathedrale von Worcester, Munich, 2000 ; Rainer Mentel, Building Scotland's Church. Das erste Jahrhundert schottisher Kirchenbaukunst 1125-1200, Weimar, 2002 et Frank Druffner, Der Chor der Kathedral von Canterbury. Architektur und Geschichte bis 1220, Egelsbach, 1994.

\section{INDEX}

Index géographique : Espagne, France, Royaume-Uni, Belgique, Allemagne, Europe centrale, Europe orientale

Keywords : Gothic architecture, research, historiography, archaeology, heritage, nationalism, reconstruction

Mots-clés : architecture gothique, recherches, historiographie, archéologie, patrimonialisation, nationalisme, reconstruction

Index chronologique : 1100, 1200, 1300, 1400, 1500, 1900, 2000

\section{AUTEURS}

\section{PHILIPPE ARAGUAS}

Philippe Araguas a été conservateur du patrimoine avant d'être professeur à l'université Michel de Montaigne de Bordeaux. Sa thèse portait sur « brique et architecture dans l'Espagne médiévale, $\mathrm{XII}^{\mathrm{e}}-\mathrm{XV}^{\mathrm{e}}$ siècles ».

\section{KLÁRA BENEŠOVSKÁ}

Klára Benešovská, a étudié le rôle des maîtres français dans l'architecture en Bohème. Elle dirige depuis 2003 le Département d'art médiéval de l'Institut d'histoire de l'art à Prague et elle a édité King John of Luxembourg and the art of his era, 1296-1346 (1998).

\section{THOMAS COOMANS}

Thomas Coomans est professeur à l'Université Libre de Bruxelles (VUB). Il est l'auteur de synthèses sur l'architecture des ordres mendiants (2001) et des cisterciens (2005) dans les anciens Pays-Bas.

\section{PETER DRAPER}

Peter Draper, actuellement professeur invité au Birkbeck College (University of London), a été président de la Society of Architectural Historians of Great Britain. Il vient de publier The Formation of English Gothic: Architecture and Identity, 1150-1250.

\section{FRANCESCA ESPAÑOL}

Francesca Español, professeur à l'Université de Barcelone, spécialiste des arts en Catalogne au Moyen Âge, a publié en 2002 un important ouvrage, El gotico catalan.

\section{BRUNO KLEIN}

Bruno Klein est professeur à la Technische Universität de Dresde et membre de l'École de l'érudition en réseau. Auteur d'une thèse sur les débuts de l'architecture gothique en France, il a édité Stilfragen zur Kunst des Mittelalters eine Einführung (2006). 


\section{DANY SANDRON}

Dany Sandron, professeur d'histoire de l'art à l'Université Paris-IV Sorbonne, est directeur du Centre André Chastel. Il a étudié les édifices gothiques du nord de la France et dirige la collection «Les Monuments de la France gothique » aux éditions Picard. 